\title{
Dynamiques temporelles de la diversité variétale du manioc (Manihot esculenta Crantz) dans deux bassins de culture en République du Congo
}

\author{
Françoise Romaine Otabo ${ }^{1, *}$, Vanesse Labeyrie ${ }^{2}$, Marie-France Duval ${ }^{3}$, Joseph Mabanza ${ }^{4}$ \\ et Fidèle Mialoundama ${ }^{5}$ \\ ${ }^{1}$ Institut national de recherche agronomique (IRA),BP 2499 IRA, Brazzaville, République du Congo \\ 2 CIRAD, UPR GREEN, 34398 Montpellier, France \\ 3 CIRAD, UMR AGAP, 34398 Montpellier, France \\ ${ }^{4}$ Centre de recherches sur l'amélioration génétique des plantes, BP 2499 CERAG/DGRST, Brazzaville, République du Congo \\ 5 Université Marien Ngouabi, BP 69, Brazzaville, République du Congo
}

\begin{abstract}
Résumé - Le manioc constitue l'aliment de base de la population congolaise. Il est majoritairement cultivé au sein d'exploitations familiales pour lesquelles la diversité variétale est un facteur majeur d'adaptation aux changements. Pourtant, la façon dont les productrices mobilisent cette diversité au cours du temps est méconnue. Cette étude caractérise donc les dynamiques de la diversité cultivée dans deux bassins de production, entre 2007 et 2012, à partir d'enquêtes auprès de 300 ménages. La diversité variétale des exploitations a été caractérisée à partir d'inventaires au champ basés sur les noms vernaculaires. Le niveau de diversité des exploitations, des villages et des sites, ainsi que la composition des portefeuilles variétaux, ont été comparés entre les deux années. De plus, la comparaison qualitative de ces inventaires avec ceux des années 1970 a permis d'établir quelles variétés étaient cultivées aux deux époques. Enfin, les causes d'abandon et d'adoption des variétés évoquées par les productrices lors des enquêtes ont été synthétisées. Le niveau de diversité est resté stable entre 2007 et 2012 ; en revanche, les portefeuilles variétaux des villages ont subi des transformations importantes et l'intensité de ces transformations varie entre les villages. À plus long terme (40 ans), on observe que certaines variétés ont été maintenues malgré les changements ayant touché les systèmes agricoles. L'influence des différents processus impliqués dans ces dynamiques est discutée.
\end{abstract}

Mots clés : diversité variétale / manioc / exploitation familiale / dynamique temporelle / République du Congo

\begin{abstract}
Temporal dynamics of cassava (Manihot esculenta Crantz) varietal diversity in two production basins in the Republic of the Congo. Cassava is the staple food of the Congolese population. It is mostly grown in family farms where varietal diversity is a major factor of adaptation to changes. However, the way producers manage this diversity over time is unknown. This study characterizes the dynamics of cassava diversity in two production areas between 2007 and 2012, based on surveys of 300 households. Farms varietal diversity was characterized from field inventories based on vernacular names. Diversity levels of farms, villages and sites and composition of varietal portfolios were compared between the two years. Moreover, the qualitative comparison of these inventories with those of the 1970s made it possible to establish which varieties were cultivated in both periods. Finally, the causes of abandon and adoption of varieties reported by producers were synthesized. Diversity levels of sites and villages remained stable between 2007 and 2012, but villages' varietal portfolios have undergone major transformations and the intensity of these changes varies among them. At a longer timescale (40 years), some varieties were maintained despite changes in agricultural systems. The influence of the different processes involved in these dynamics is discussed.
\end{abstract}

Keywords: agrobiodiversity / cassava / family farming / temporal dynamics / Republic of the Congo

\footnotetext{
* Auteur de correspondance : otabo.francoise@gmail.com
} 


\section{Introduction}

$\mathrm{Au}$ Congo, la culture du manioc constitue l'activité principale pour près de $98 \%$ des agriculteurs (FAO, 2006). L'espèce est majoritairement cultivée par les femmes au sein d'exploitations familiales. Cependant, la culture du manioc dans ces exploitations est soumise à d'importants changements environnementaux qui limitent son développement et menacent la durabilité des systèmes de culture. Les changements environnementaux ayant affecté le plus fortement la culture du manioc dans le pays au cours des dernières décennies sont de nature biotique. L'expansion de la mosaïque africaine du manioc, de la bactériose vasculaire (Xanthomonas sp.) et de la striure brune constituent une menace importante pour la production de manioc au Congo (Legg et al., 2006, 2011; Mvila et al., 2003).

La durabilité et l'adaptation des systèmes agricoles familiaux dépendent fortement de la diversité qui y est cultivée (Altieri, 1999). La diversification des espèces et des variétés cultivées permet notamment de limiter l'impact des pathogènes et des ravageurs des cultures (Letourneau et al., 2011; Ratnadass et al., 2012). Au Congo, la diversité variétale du manioc maintenue dans les exploitations familiales est mal connue, et de ce fait peu valorisée. Cette espèce originaire du bassin amazonien a été introduite en Afrique au XVI ${ }^{\mathrm{e}}$ siècle par les Portugais (Olsen et Schaal, 1999). Les seules informations disponibles sur sa diversité sont issues d'inventaires in situ basés sur les noms vernaculaires, et font état d'une grande diversité de variétés nommées et de différences de composition variétale entre régions (Kombo et al., 2012; Otabo et al., 2016).

Les pratiques de gestion de la diversité variétale du manioc par les productrices sont un levier majeur de l'adaptation des exploitations familiales congolaises aux changements socio-économiques et agroécologiques auxquels elles sont soumises. Pourtant, ces pratiques n'ont pas été documentées. L'agrobiodiversité étant un facteur d'adaptation important des exploitations familiales, nous faisons l'hypothèse que les producteurs font évoluer leur portefeuille variétal au cours du temps pour s'adapter aux nouvelles contraintes et opportunités auxquelles ils font face. Cependant, le renouvellement des variétés dans les exploitations est fortement contraint par le faible taux de multiplication de cette espèce, reproduite par bouturage. Mieux comprendre les pratiques de gestion de la diversité variétale des exploitations familiales et leurs déterminants permettrait de mieux cibler les actions d'appui aux producteurs, notamment pour améliorer leur accès à du matériel végétal adapté et diversifié.

Cette étude vise donc à caractériser les dynamiques temporelles du manioc dans les exploitations agricoles familiales congolaises au travers de trois questions:

- le niveau de diversité et la composition des portefeuilles variétaux des exploitations varient-ils au cours du temps?

- ces dynamiques temporelles diffèrent-elles entre les zones géographiques?

- quels sont les facteurs expliquant l'adoption ou l'abandon des variétés?

Pour cela, la diversité des portefeuilles variétaux des exploitations de deux bassins de production a été comparée entre 2007 et 2012, et les raisons d'abandon ou d'adoption des

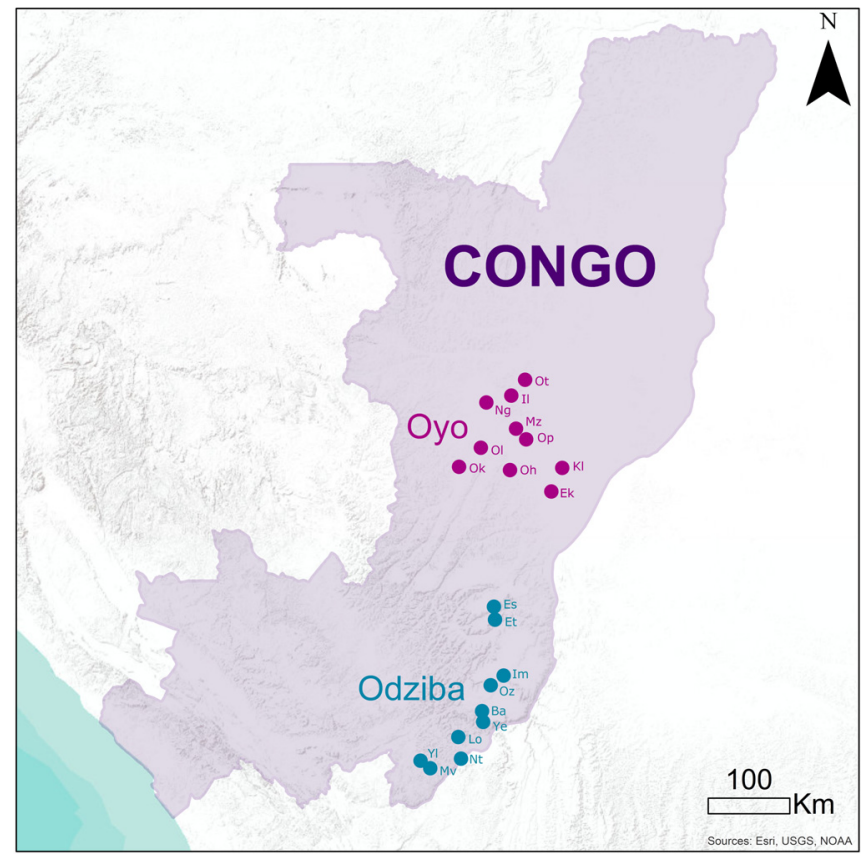

Fig. 1. Localisation des sites d'étude et abréviations des noms des villages. Site d'Odziba (Bambou mingali: BA; Essoua: ES; Etsouali: ET; Imbama: IM; Loumou: LO; Mvoulou mamba Sud : MV; Ntoula : NT; Odziba : OZ; Yie: YE; Yala mvounga : YL). Site d'Oyo (Ekongo: EK; Illebou Ondongo: IL, Koli: KL, Moundzeli: MZ; Ngoko: NG; Ohouri: OH; Okouesse: OK; Olongonen: OL; Opokania: OP; Otende: OT).

Fig. 1. Location of study sites.

différentes variétés ont été analysées. D'autre part, le maintien des variétés sur le long terme a été évalué en comparant ces inventaires avec ceux effectués dans les années 1970. Les résultats de cette étude dressent des pistes pour des travaux de recherche futurs. La comparaison des dynamiques temporelles de la diversité entre les deux bassins de production étudiés et entre villages au sein des bassins permet notamment de proposer des hypothèses à explorer concernant les facteurs impliqués dans les dynamiques de la diversité.

\section{Matériel et méthodes}

\subsection{Sites d'étude}

Deux sites de production ont été choisis en raison de l'importance des activités de production et de transformation du manioc dans ces zones (Fig. 1). Ces deux sites présentent des conditions socio-économiques, culturelles et agroécologiques contrastées. Le site d'Oyo est situé au Nord du Congo sur l'axe routier de la nationale (N2), à environ $500 \mathrm{~km} \mathrm{de}$ Brazzaville. Il se situe en zone forestière tropicale humide et présente un climat subéquatorial (moyennes annuelles: température $23-26^{\circ} \mathrm{C}$, précipitations $1600-1800 \mathrm{~mm}$; FAO, 2005). Le site d'Odziba est subdivisé en deux sous-zones (Sud et Nord) présentant des caractéristiques agroécologiques différentes. La partie Nord est située en zone de savane à environ $100 \mathrm{~km}$ de Brazzaville sur la route nationale 2, caractérisée par un climat similaire à celui de la zone d'Oyo. La partie Sud, en zone forestière, est située à environ $75 \mathrm{~km}$ de 
Brazzaville sur la route nationale 1 et est caractérisée par un climat tropical (moyennes annuelles : températures $21-27^{\circ} \mathrm{C}$, pluviométrie 1200-1700 mm; FAO, 2005). Le lingala est la langue dominante à Oyo et dans la zone nord du site d'Odziba, tandis que le kituba est majoritaire dans la zone sud d'Odziba. À Oyo, l'ethnie Bochis est dominante, alors qu'à Odziba, les Laris sont dominants. Dans les deux sites, le manioc est majoritairement cultivé dans des systèmes agricoles familiaux, non mécanisés et à bas niveaux d'intrants, en savane dans le site d'Odziba et en forêt à Oyo. Les boutures sont plantées après défriche et brûlis, disposées à plat ou sur buttes, en association avec diverses espèces potagères, fruitières, ou céréalières. Les usages du manioc dans les deux sites sont similaires, les racines et les feuilles sont utilisées dans l'alimentation humaine et dans une moindre mesure pour l'alimentation du bétail. Les feuilles sont transformées en saka-saka (légume) et les tubercules sont principalement transformés en chikwangue (pâte de manioc), en farine (foufou) et en boisson (en mélange avec du maïs).

\subsection{Collecte des données}

Des inventaires des variétés de manioc cultivées en 2007 et en 2012 ont été réalisés dans les deux sites. Les enquêtes ont été menées dans dix villages par site, et auprès de 15 productrices dans chaque village, soit un total de 20 villages et 300 exploitations. Les ménages interrogés ont été sélectionnés sur la base du volontariat et de la disponibilité des productrices. Les inventaires ont été réalisés au champ avec chaque productrice, sur la base des noms vernaculaires des variétés utilisées par cette dernière. Le terme variété est donc entendu comme un ensemble de plantes que les productrices regroupent dans une catégorie identifiée par un nom propre car elles présentent des caractéristiques semblables de leur point de vue (Pinton et Emperaire, 2001). Chaque variété représente donc ce que l'on peut qualifier de type nommé (Caillon et Lanouguère-Bruneau, 2005). Au cours de ces enquêtes, il a été demandé aux productrices de citer les raisons d'adoption des différentes variétés présentes dans leur champ, et les causes d'abandon de celles qu'elles ont cessées de cultiver.

D'autre part, des données provenant d'inventaires variétaux effectués dans les deux sites dans les années 1970 ont été obtenues (Mabanza, 1992). Cependant, les protocoles utilisés pour la collecte de données n'étaient pas précisés dans les rapports d'enquêtes ; ces données permettent donc uniquement de déterminer si certaines variétés présentes dans les années 1970 dans la zone ont été maintenues, mais elles ne permettent pas de connaître la fréquence des variétés ni d'effectuer des comparaisons approfondies de la composition variétale entre les deux époques.

\subsection{Analyses des données}

Pour pouvoir comparer les portefeuilles variétaux des différentes unités d'étude (champ, village, site), une homogénéisation de l'ensemble des noms bruts inventoriés dans chaque village a été réalisée pour s'affranchir des variations de prononciation entre les personnes interrogées et des erreurs de retranscription.
La diversité variétale de chacun des sites, mais aussi de chacun des villages au sein des sites, a été comparée entre 2007 et 2012. Deux types d'analyses ont été effectuées pour cela : - une comparaison quantitative du niveau de diversité entre 2007 et 2012 basée sur deux indices : la richesse $(S)$, qui correspond au nombre de variétés cultivées dans l'unité d'étude; l'indice composite de Shannon $(H)$, qui tient compte à la fois du nombre de variétés cultivées et du nombre d'exploitations où chacune d'elles est cultivée (Eq. 1 ; Shannon, 1948);

- une comparaison de la composition variétale tenant compte de l'identité des variétés, basée sur des analyses multivariées.

L'ensemble des analyses a été réalisé sous $\mathrm{R}$ version 3.2.3 (R Core Team, 2015; Tab. S1).

Afin de caractériser l'évolution du niveau de diversité, le nombre de variétés cultivées par exploitation, par village, et par site, a été comparé entre 2007 et 2012 . Premièrement, la richesse variétale de chaque exploitation $\left(S_{a}\right)$ a été calculée à partir des inventaires pour chacune des deux années d'étude. Sur cette base, la richesse moyenne des exploitations (nombre moyen de variétés cultivées par exploitation) et son écart-type ont été calculés pour chaque village et pour chaque site, pour chacune des deux années. Deuxièmement, la richesse variétale a été calculée pour chaque site $\left(S_{s}\right.$; nombre total de variétés différentes inventoriées dans le site) et pour chaque village dans chacun des sites $\left(S_{v}\right.$; nombre total de variétés différentes inventoriées dans le village). La significativité de l'évolution de $S_{S}$ dans chaque site et de $S_{v}$ dans chaque village entre 2007 et 2012 a été déterminée par des tests de randomisation implémentés dans le package $\mathrm{R}$ rich (Rossi, 2011).

L'indice composite de diversité de Shannon, tenant compte à la fois de la richesse et de la distribution de fréquence des différentes variétés, a été calculé pour chaque site $\left(H_{S}\right)$ et chaque village $\left(H_{v}\right)$ dans chacun des sites suivant la formule :

$$
H=-\sum_{i=1}^{s} p_{i} \log p_{i} \text {. }
$$

Avec $p_{i}$ la proportion de la variété $i$ dans l'unité d'étude considérée (village ou site).

Les valeurs de $H_{s}$ dans chaque site et $H_{v}$ dans chaque village ont été comparées entre les deux années en construisant un intervalle de confiance à $95 \%$ par bootstrapping (2000 permutations) avec le package R simboot (Scherer et Pallmann, 2014).

Afin de tester si la composition des portefeuilles variétaux des sites ainsi que celle des villages au sein de chaque site différaient significativement entre 2007 et 2012, une analyse de variance multivariée sur matrice de distance, implémentée dans le package $\mathrm{R}$ vegan, a été effectuée (perMANOVA; Oksanen et al., 2013). Cette méthode d'analyse a permis de tester si le facteur année explique une part significative de la variance de composition variétale des sites et des villages. Afin de déterminer quelles variétés étaient à l'origine des différences détectées par la perMANOVA, des tests exacts de Fisher ont ensuite été effectués pour tester si l'abondance de chacune des variétés dans chaque site et chaque village avait varié significativement entre les deux années. 
Tableau 1. Indices de diversité variétale à Oyo (haut) et Odziba (bas). Indices de Shannon et richesse à l'échelle du village $\left(H_{v}\right.$ et $\mathrm{S}_{\mathrm{v}} ; n=15$ agriculteurs, $N=10$ villages), et du site $\left(H_{s}\right.$ et $\mathrm{S}_{\mathrm{s}} ; \mathrm{N}=10$ villages).

Table 1. Indices of varietal diversity in Oyo (up) and Odziba (down). Shannon indices and varietal richness at the village $\left(\mathrm{H}_{\mathrm{v}}\right.$ and $\mathrm{S}_{\mathrm{v}}, \mathrm{n}=15$ farmers, $\mathrm{N}=10$ villages), and site scale $\left(\mathrm{H}_{\mathrm{s}}\right.$ and $\mathrm{S}_{\mathrm{s}} ; \mathrm{N}=10$ villages).

\begin{tabular}{|c|c|c|c|c|c|}
\hline Année & $S_{a} \mathrm{M}(\mathrm{ET})$ & $S_{v} \mathrm{M}(\mathrm{ET})^{1}$ & $H_{v} \mathrm{M}(\mathrm{ET})^{1}$ & $S_{s}^{2}$ & $H_{s}^{3}$ \\
\hline \multicolumn{6}{|l|}{ Oyo } \\
\hline 2007 & $2,7(1.0)$ & $9,6(3,9)^{\mathrm{a}}$ & $1,9(0,3)^{\mathrm{a}}$ & $25^{\mathrm{a}}$ & $2,5^{\circ}$ \\
\hline \multicolumn{6}{|l|}{ Odziba } \\
\hline 2007 & $2,3(1,2)$ & $6,3(1,8)^{\mathrm{a}}$ & $1,4(0,4)^{\mathrm{a}}$ & $35^{\mathrm{a}}$ & $2,8^{\circ}$ \\
\hline
\end{tabular}

$\mathrm{M}$ : moyenne, ET : écart-type. ${ }^{1,2,3}$ Les lettres minuscules en exposant $(\mathrm{a}, \mathrm{b})$ indiquent la significativité des différences $\left({ }^{1}\right.$ test de Wilcoxon : $P<0,05 ;{ }^{2}$ test par randomisation ${ }^{3}$ intervalle de confiance à $95 \%$ par bootstrap).

Afin d'identifier les principales raisons d'adoption ou d'abandon des variétés, la proportion de productrices ayant cité chaque critère pour les principales variétés a été calculée dans chaque village (variétés cultivées dans plus de 10 exploitations). La moyenne sur l'ensemble des villages au sein de chaque site a été calculée pour chacune des deux années.

\section{Résultats}

\subsection{Niveau de diversité variétale}

$\mathrm{Au}$ total, 59 variétés nommées ont été recensées dans les deux sites en 2007 (Oyo: 25, Odziba: 35-1 variété en commun) et 100 en 2012 (Oyo : 54, Odziba : 48-2 variétés en commun). Quarante-quatre variétés ont été recensées à la fois en 2007 et 2012. Les deux sites présentent des compositions variétales totalement différentes puisqu'une seule variété était commune aux deux sites en 2007 , et deux variétés étaient communes en 2012.

À Oyo, le test de randomisation montre que la richesse totale $\left(S_{S}\right)$ est significativement plus faible en 2007 (25 variétés) qu'en 2012 (54 variétés, test de randomisation: $P<0,01$ ), tandis qu'aucune différence significative n'est observée pour Odziba ( 35 variétés en 2007,48 en $2012 ; P>0,05$ ) (Tab. 1). En revanche, aucune différence significative de l'indice composite de diversité $H_{S}$ n'a été détectée entre 2007 et 2012 dans aucun des deux sites (test de randomisation: $P>0,05$ ).

Les valeurs de $S_{v}$ et $H_{v}$ ne présentent pas d'évolution significative entre 2007 et 2012 dans la plupart des villages de chacun des deux sites. En effet, $S_{v}$ a augmenté significativement dans seulement deux villages à Oyo (EK et OL, test de randomisation: $P<0,05)$ et à Odziba ( $\mathrm{LO}$ et $\mathrm{YL}$, test de randomisation: $P<0,05)$, tandis que $H v$ a augmenté significativement dans un village à Oyo $(\mathrm{OL})$ et trois à Odziba (LO, YL et ET). Enfin, le nombre de variétés cultivées en moyenne par agriculteur $S_{a}$ ainsi que son écart-type sont légèrement plus élevés en 2012 qu'en 2007 dans les deux sites.

\subsection{Composition variétale}

Les différences de composition variétale entre 2007 et 2012 pour chacun des sites ne sont pas significatives (perMANOVA: $P>0,05$; Tab. 2). Cependant, l'abondance de huit variétés ( $14 \%$ des variétés cultivées) à Oyo et neuf à
Tableau 2. Différences de composition variétale entre années à l'échelle des sites et des villages: résultats des analyses de variance multivariées sur matrice de distance (Adonis).

Table 2. Differences in varietal composition between years at the scale of the sites and the villages: results of the multivariate analyzes on distance matrix (Adonis).

\begin{tabular}{|c|c|c|c|}
\hline \multirow[b]{2}{*}{ Village } & \multicolumn{3}{|c|}{ Adonis } \\
\hline & $\mathrm{F}$ & $R^{2}$ & $P$ \\
\hline Oуо & 1,4 & 0,07 & 0,174 \\
\hline \multicolumn{4}{|l|}{ Villages } \\
\hline EK & 26,1 & 0,48 & $0,001^{* * *}$ \\
\hline IL & 7,5 & 0,21 & $0,001^{* * *}$ \\
\hline KL & 12,4 & 0,31 & $0,001^{* * *}$ \\
\hline $\mathrm{MZ}$ & 15,2 & 0,35 & $0,001^{* * *}$ \\
\hline NG & 3,3 & 0,11 & $0,019^{*}$ \\
\hline $\mathrm{OH}$ & 3,4 & 0,11 & $0,001^{* * *}$ \\
\hline OK & 3,6 & 0,11 & $0,021^{*}$ \\
\hline $\mathrm{OL}$ & 4,2 & 0,13 & $0,003 * *$ \\
\hline OP & 4,5 & 0,14 & $0,001^{* * *}$ \\
\hline OT & 11,6 & 0,29 & $0,001^{* * *}$ \\
\hline Odziba & 0,4 & 0,02 & 0,87 \\
\hline \multicolumn{4}{|l|}{ Villages } \\
\hline $\mathrm{BA}^{\mathrm{n}}$ & 15,8 & 0,36 & $0,001 * * *$ \\
\hline $\mathrm{ES}^{\mathrm{n}}$ & 18,3 & 0,40 & $0,001^{* * *}$ \\
\hline $\mathrm{ET}^{\mathrm{n}}$ & 20,2 & 0,42 & $0,001 * * *$ \\
\hline $\mathrm{IM}^{\mathrm{n}}$ & 8,2 & 0,23 & $0,001^{* * *}$ \\
\hline $\mathrm{OZ}^{\mathrm{n}}$ & 35,0 & 0,56 & $0,001 * * *$ \\
\hline$Y^{n}$ & 20,0 & 0,42 & $0,001^{* * *}$ \\
\hline $\mathrm{LO}^{\mathrm{s}}$ & 6,2 & 0,18 & $0,002 * *$ \\
\hline $\mathrm{MV}^{\mathrm{s}}$ & 17,1 & 0,38 & $0,001 * * *$ \\
\hline $\mathrm{NT}^{\mathrm{s}}$ & 23,4 & 0,45 & $0,001 * * *$ \\
\hline $\mathrm{YL}^{\mathrm{s}}$ & 7,1 & 0,20 & $0,001^{* * *}$ \\
\hline
\end{tabular}

$\mathrm{F}$ : pseudo-F ratio, $R^{2}$ : coefficient de détermination, $P: P$-value; ***: $P \leq 0,001 ;{ }^{* *}: P \leq 0,01 ;{ }^{*}: P \leq 0,05 ;{ }^{\mathrm{n}}$ : Odziba-Nord ${ }^{\mathrm{s}}$ : Odziba-Sud.

Odziba $(15 \%)$ a augmenté significativement entre 2007 et 2012 , tandis que celle de sept variétés $(12 \%)$ à Oyo et trois à Odziba $(5 \%)$ a diminué (tests exacts de Fisher: $P<0,05$; 

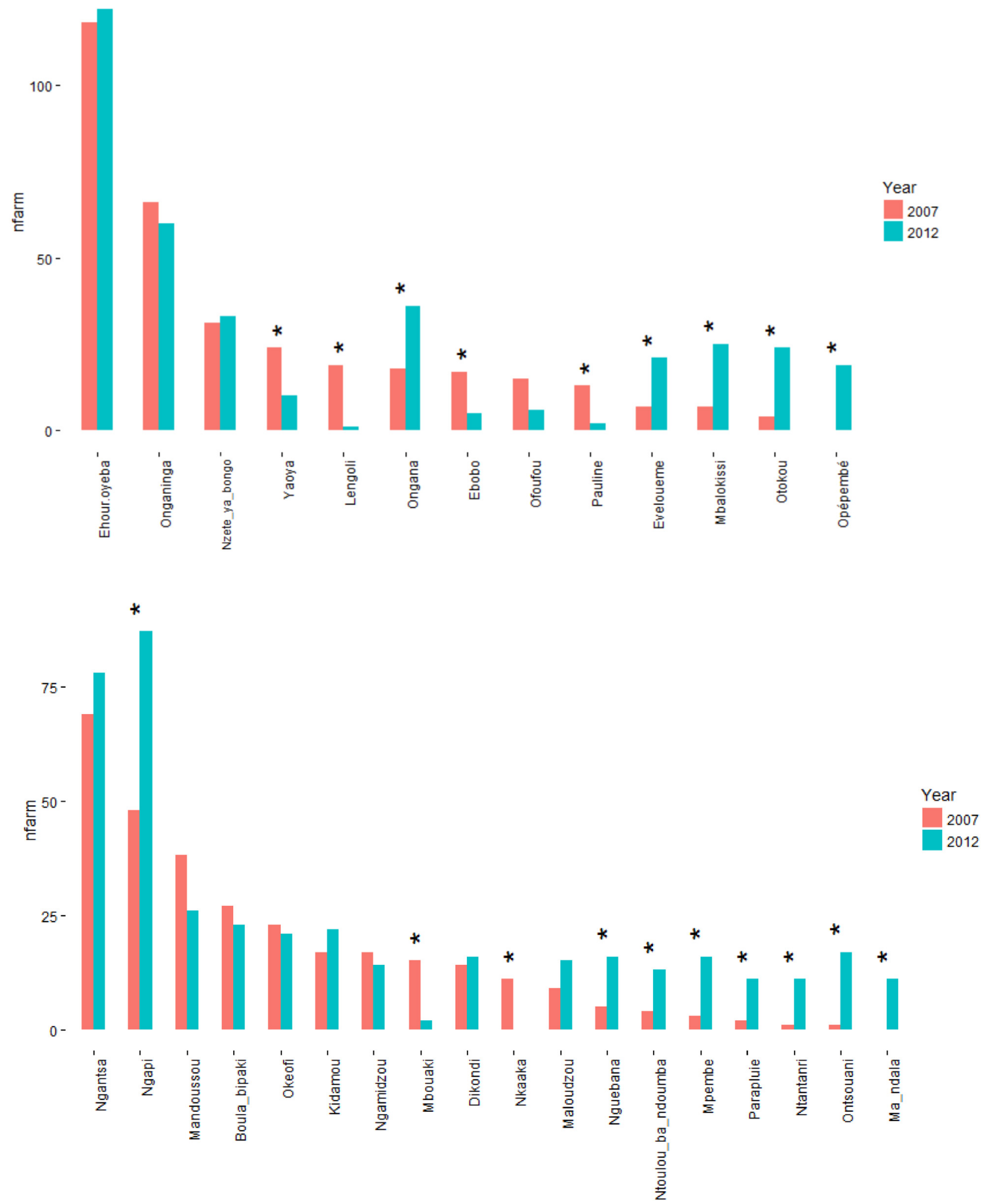

Fig. 2. Abondance des principales variétés en 2007 et 2012 à Oyo (haut) et Odziba (bas). (test exact de Fisher, $P<0,05:{ }^{*}$ ). Fig. 2. Abundance of the main varieties in 2007 and 2012 in Oyo (up) and Odziba (down). (Fisher's exact test, $\mathrm{P}<0.05$ : *).

Tab. S2). La plupart des variétés ne montrent pas de variation significative d'abondance (tests exacts de Fisher: $P>0,05$ ). À Oyo, l'abondance des deux variétés les plus cultivées (Ehour
Oyeba, Onganinga) reste stable entre les deux années (Fig. 2). C'est plutôt l'abondance des variétés intermédiaires qui varie significativement, ainsi que celle de quelques variétés très rares 


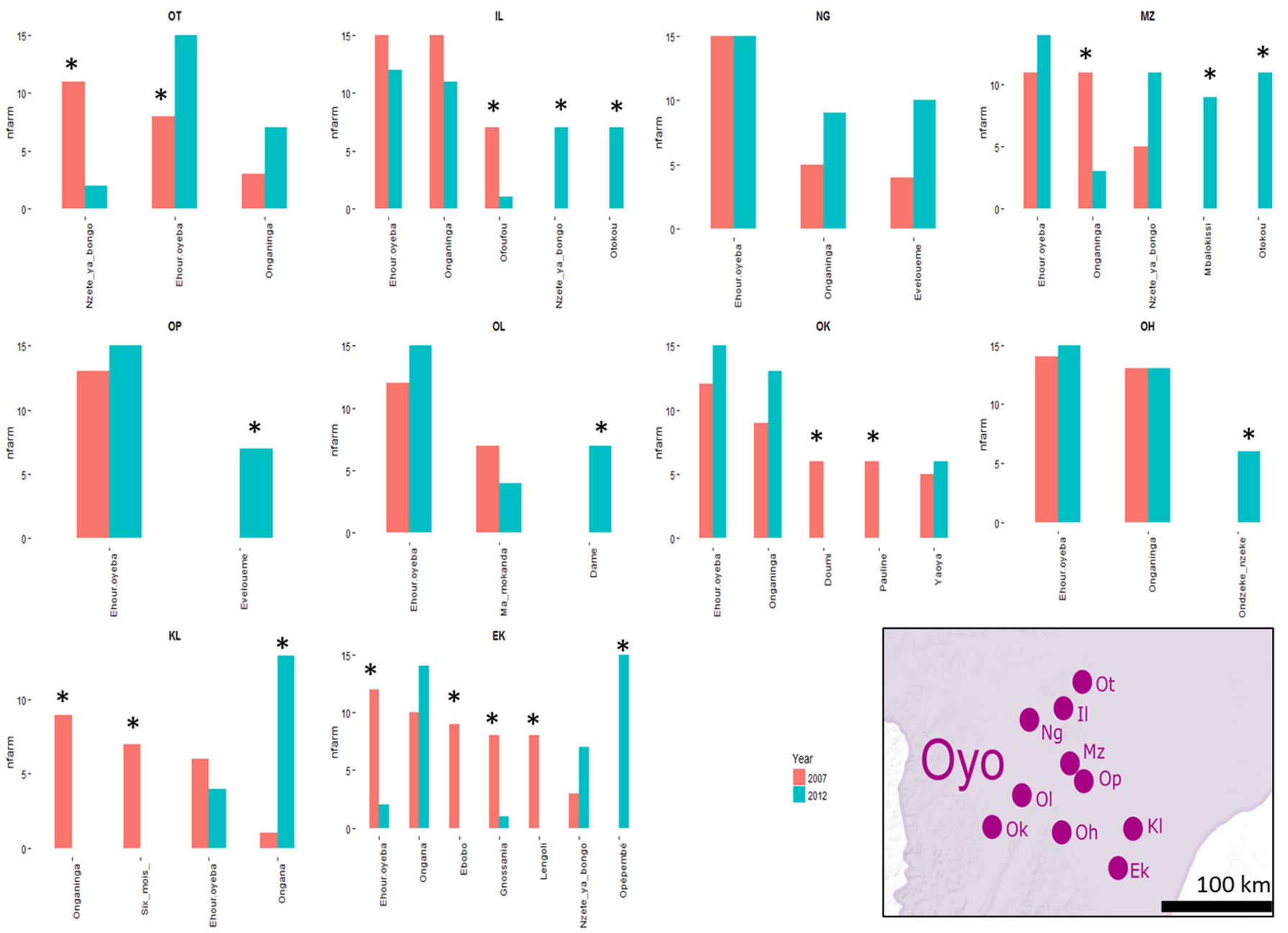

Fig. 3. Abondance des principales variétés en 2007 et 2012 dans chaque village à Oyo (haut) et Odziba (bas). (test exact de Fisher, $P<0,05: *$ ). Fig. 3. Abundance of the main varieties in 2007 and 2012 in each village in Oyo (up) and Odziba (down). (Fisher's exact test, $\mathrm{P}<0.05$ : *).

ou absentes en 2007, comme Opépembé. À Odziba, en revanche, on observe une forte augmentation de l'abondance de la variété $N g a p i$, qui était déjà la $2^{\mathrm{e}}$ variété la plus abondante après Ngantsa en 2007, et passe au premier rang en 2012. Le nombre de variétés rares augmente fortement en 2012 dans les deux sites (Tab. S2).

À l'échelle des villages, en revanche, la composition variétale diffère significativement entre 2007 et 2012 (perMANOVA: $P<0,05$; Tab. 2), mais ces différences sont plus marquées dans certains villages. À Oyo, les villages situés au sud-est de la zone d'étude et éloignés de la route N2 (EK et $\mathrm{KL}$ ) ont subi de fortes transformations de leur composition, comme le montre la part importante de variation expliquée par l'année pour ces sites $\left(R^{2}>0,3\right)$. En revanche, les variétés dont l'abondance varie significativement entre les deux années ne sont pas les mêmes dans ces deux villages (tests exacts de Fisher: $P<0,05)$. Notamment, l'abondance d'Ehour Oyeba à EK, où elle était dominante en 2007, diminue fortement, tandis qu'elle reste dominante dans la plupart des villages de la zone en 2012 (Fig. 3). À KL, Ongana fait son apparition et devient dominante. Les villages de MZ au centre de la zone d'Oyo et d'IL et OT au nord, tous trois assez proches de la N2, ont également subi d'assez fortes modifications de leurs cortèges
$\left(R^{2}>0,20\right)$. À MZ, l'abondance d'Onganinga diminue fortement alors qu'elle était co-dominante en 2007. À OT, l'abondance d'Ehour Oyeba augmente fortement et elle devient dominante, tandis que Nzete ya bongo, qui était dominante en 2007, disparaît quasiment (tests exacts de Fisher: $P<0,05)$. Le reste des villages, situés majoritairement à l'ouest et éloignés de la N2 à l'exception d'OP, montrent moins de modifications de leur composition entre les deux années $\left(R^{2}<0,15\right)$. À NG, seule une variété disparaît, et dans les villages d'OK, $\mathrm{OL}, \mathrm{OH}$ et $\mathrm{OP}$, seules quelques variétés modérément abondantes apparaissent ou disparaissent en 2012 (tests exacts de Fisher: $P<0,05$ ).

À Odziba, on distingue clairement deux groupes de villages présentant des compositions totalement différentes, le premier groupe correspondant aux villages du nord de la zone (ES, ET, IM, OZ, BA, YE) et le second à ceux du sud (LO, NT, MV, YL). La composition des cortèges variétaux des villages du nord est assez homogène, avec deux variétés dominantes (Ngantsa et Ngapi), tandis que dans les villages du sud, on observe une dominance moins marquée et des compositions qui diffèrent davantage entre villages. À Odziba-Nord, la composition des cortèges variétaux des villages est plus similaire en 2012 qu'en 2007, exception faite du village OZ, et 

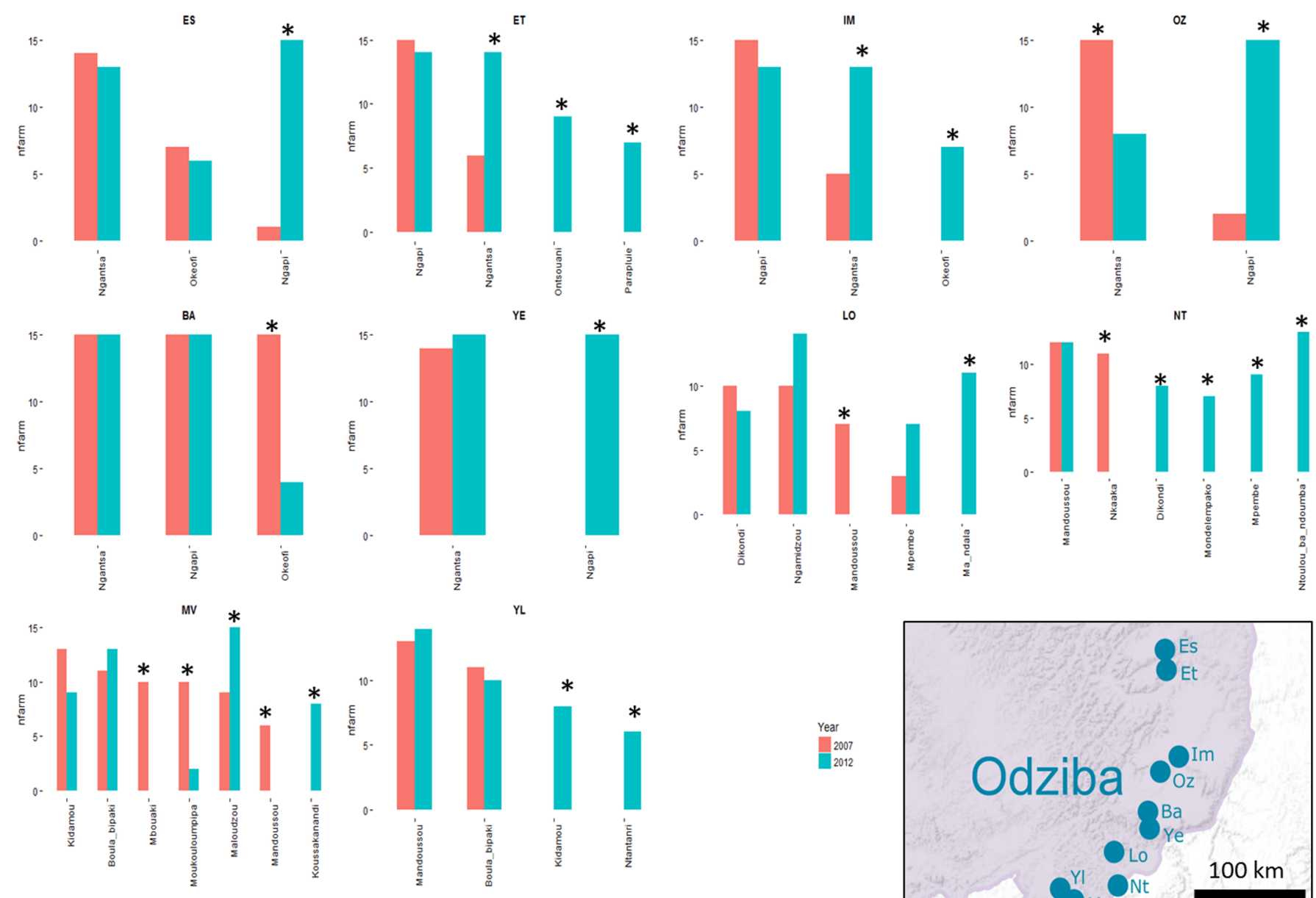

Fig. 3. Continued

on observe des modifications importantes de composition dans tous les villages $\left(R^{2}>0,23\right)$. L'abondance de Ngapi augmente fortement dans les trois villages où elle n'était pas dominante en 2007 (ES, OZ et YE), et le même phénomène est observé pour Ngantsa, dont l'abondance augmente dans les villages d'ET et d'IM (tests exacts de Fisher: $P<0,05$ ). De ce fait, la composition des cortèges variétaux des villages s'uniformise en 2012 avec deux variétés co-dominantes dans les cortèges variétaux: Ngantsa et Ngapi. Seul le village d'Odziba se distingue, car l'abondance de Ngantsa y diminue significativement en $2012(P<0,05)$. À Odziba-Sud, les villages de MV et NT montrent des modifications importantes de leurs cortèges variétaux $\left(R^{2}>0,37\right)$. Maloudzou devient notamment dominante en 2012 à MV. Les villages de LO et YL subissent une moindre modification de leur composition variétale $\left(R^{2}<0,21\right)$.

La comparaison des inventaires réalisés en 2007 et 2012 avec ceux des années 1970 montre que 21 variétés parmi les 42 relevées à Oyo dans les années 1970, et 11 sur les 41 relevées à Odziba, sont toujours présentes soit en 2007, soit en 2012, soit aux deux dates. De plus, les variétés majeures recensées à Oyo étaient déjà cultivées dans les années 1970 (Ehour Oyeba, Onganina, Nzete ya bongo). À Odziba, Ngapi était déjà présente ainsi que Moundelepako. En revanche, Ngantsa, qui était dominante en 2012 et 2007, n'avait pas été répertoriée dans les années 1970, et c'est également le cas pour Mandoussou.

\subsection{Raisons d'adoption et causes d'abandon des variétés}

La productivité élevée, la résistance aux maladies, et la qualité du produit fini sont les principaux critères d'adoption cités par l'ensemble des productrices dans les deux sites (moyenne sur les deux sites et les deux années : 100\% des réponses, Fig. 4). Viennent ensuite la disponibilité du matériel végétal $(96 \%)$, la conservation (94\%), et la précocité (92\%). Il y a peu de différences entre les deux sites, à part pour la précocité et la disponibilité du matériel végétal qui sont davantage citées à Oyo ( $84 \%$ et $99 \%$ respectivement) qu'à Odziba (60\% et $89 \%)$.

La faible productivité est la principale cause d'abandon des variétés rapportée par les productrices (moyenne sur les deux sites et les deux années: $70 \%$, Fig. 4). Les problèmes de disponibilité du matériel végétal $(64 \%)$ et de pourriture des tubercules $(48 \%)$ ont également été fréquemment cités. Les 
Raisons d'adoption - Odziba

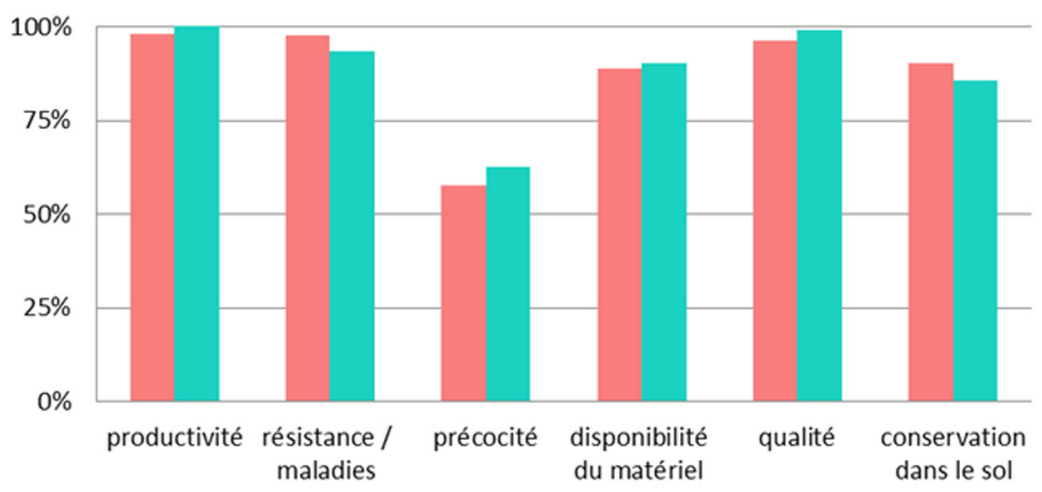

Raisons d'adoption - Oyo

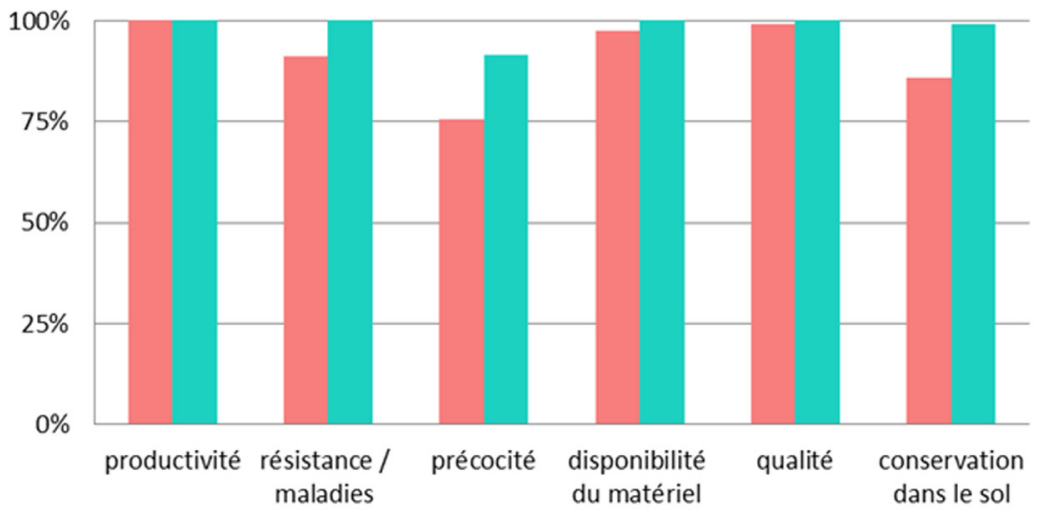

Causes d'abandon - Odziba

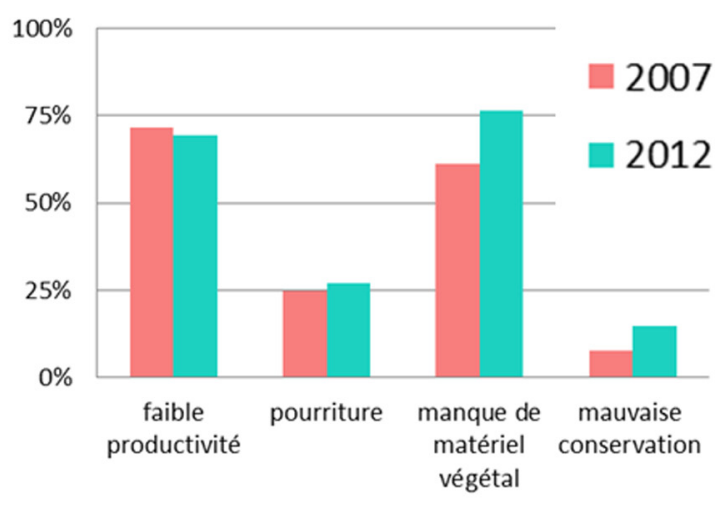

Causes d'abandon - Oyo

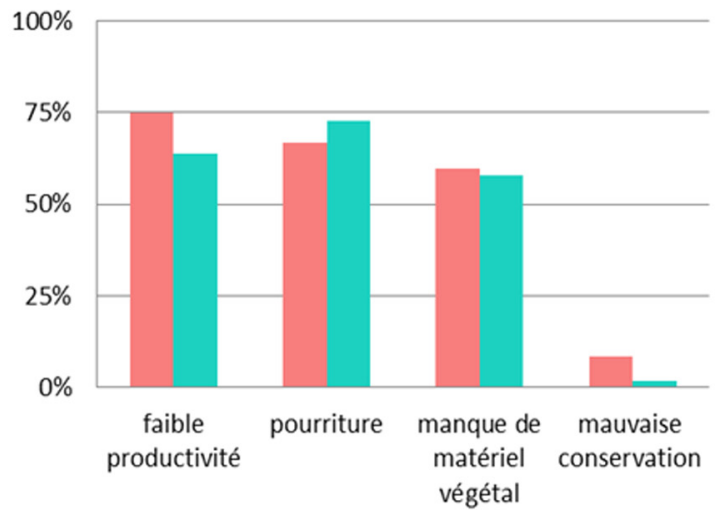

Fig. 4. Raisons d'adoption et causes d'abandon des principales variétés en 2007 et 2012. Moyenne du pourcentage de productrices ayant cité chaque raison d'adoption ou cause d'abandon.

Fig. 4. Reasons for adoption and causes of discontinuation of major varieties in 2007 and 2012. Average percentage of producers citing each reason for adoption or cause of discontinuation.

problèmes de conservation ont été peu cités $(8 \%)$. De fortes différences de fréquence de citation ont été observées entre les deux sites pour la pourriture des tubercules, qui est davantage citée à Oyo $(70 \%)$ qu'à Odziba $(26 \%)$. Le manque de matériel végétal et la mauvaise conservation sont un peu moins fréquemment cités à Oyo $(59 \%$ et $5 \%$ respectivement) qu'à Odziba $(69 \%$ et $11 \%)$.

\section{Discussion}

Nos résultats montrent que le niveau de diversité est resté assez stable entre 2007 et 2012 à l'échelle des sites et des villages, et que la composition des portefeuilles variétaux à l'échelle des sites ne varie pas significativement entre ces deux années. En revanche, les analyses à l'échelle des villages montrent que la composition des portefeuilles variétaux a subi des transformations importantes sur le pas de temps relativement court de notre étude, confirmant de précédentes observations dans la zone de Bouenza, proche du site d'Oyo (Kombo et al., 2012). Cette étude montre également qu'une part importante des variétés recensées dans les années 1970 continue d'être cultivée au sein des exploitations, et que certaines de ces variétés sont même dominantes dans certaines zones. Cela souligne l'importance des variétés locales pour les productrices, confirmant les observations de Kombo et al. (2012), qui avaient inventorié une large majorité de variétés locales dans les exploitations. Ces résultats montrent que les divers changements auxquels ont été soumis les exploitations n'ont pas conduit à la disparition des variétés locales, contrairement à ce qui a été observé dans d'autres situations, notamment face à l'introduction de variétés améliorées (Chaudhary et al., 2004; Pham et al., 2002), ou à la modification des systèmes agricoles (Peroni et Hanazaki, 2002).

Cette étude étant basée sur les noms vernaculaires, elle ne nous permet pas d'évaluer si ces dynamiques temporelles s'observent aussi au niveau de la diversité phénotypique et génétique. Les études menées sur la diversité du manioc, notamment en Océanie et en Amérique du Sud, ont montré une bonne correspondance entre diversité phénotypique et génétique et diversité nommée à l'échelle des villages (Elias et al., 2001 ; Sardos et al., 2008). En effet, les agriculteurs se basent sur les critères morphologiques pour identifier les variétés, et l'échange d'informations et de matériel végétal au sein de la communauté favorise l'adoption d'une nomenclature commune, partagée par la majorité des individus (Boster, 1986). Cependant, ces études ont également montré que bien 
que les variétés soient généralement distinctes génétiquement et phénotypiquement, une partie d'entre elles présente une diversité infra-variétale élevée. De ce fait, on peut s'attendre à ce que la diversité des noms vernaculaires à l'échelle d'un village sous-estime la diversité génétique et phénotypique. L'analyse de la diversité génétique et de sa correspondance avec la nomenclature locale permettrait de préciser si les modifications de composition des portefeuilles variétaux correspondent à des modifications de la diversité génétique, et de retracer la nature et l'origine des différentes variétés.

Nos résultats ont mis en évidence de fortes différences de dynamiques de la composition variétale entre les sites, et entre les villages dans chaque site. Ils suggèrent que les systèmes semenciers locaux sont très dynamiques, permettant aux productrices d'avoir accès à de nouvelles variétés pour s'adapter aux changements auxquels elles sont confrontées. La synthèse d'études menées sur différentes espèces et dans divers pays montre l'efficacité des systèmes semenciers locaux, favorisant la diffusion rapide de nouvelles variétés, parfois sur de longues distances (Coomes et al., 2015). Ces études montrent notamment que les agriculteurs ont fréquemment recours aux marchés pour se procurer du matériel végétal (McGuire et Sperling, 2016), et mobilisent leur réseau de relations sociales, notamment leur parentèle (Labeyrie et al., 2015). Cependant, Kombo et al. (2012) rapportent que la majorité du matériel végétal utilisé par les exploitations d'une zone proche d'Oyo est autoproduit, et que seul $6 \%$ est obtenu auprès des proches, et $0,6 \%$ sur les marchés. Ces résultats sont en contradiction avec l'ampleur des dynamiques observées dans notre étude. Une investigation approfondie des réseaux semenciers locaux et de leurs dynamiques temporelles serait donc nécessaire pour éclaircir leur rôle dans les dynamiques des portefeuilles variétaux.

Une autre source potentielle de diversité est la sélection des plantules issues de la reproduction sexuée par les productrices, comme cela a été montré en Océanie (Sardos et al., 2008) et en Amérique du Sud (Pujol et al., 2005). Dans les enquêtes menées par Kombo et al. (2012), une faible part des productrices $(11,7 \%)$ ont rapporté conserver les plantules issues de graines dans leurs champs, mais elles n'ont en revanche pas indiqué connaître des variétés issues de ce processus. Ce processus de création variétale semble donc être plutôt anecdotique, et il est donc peu plausible qu'il soit à l'origine des dynamiques observées.

Concernant les motivations des productrices pour modifier leurs portefeuilles variétaux, les enquêtes montrent que la baisse de productivité est un facteur majeur expliquant l'abandon des variétés, tandis que la productivité et la résistance aux maladies sont des critères fondamentaux dans l'adoption de nouvelles variétés. Cela suggère que les pressions biotiques sont un moteur majeur de l'évolution des portefeuilles variétaux des exploitations congolaises, confortant les observations de Kombo et al. (2012). En effet, les dégâts causés par les différents pathogènes et leur accumulation dans le matériel végétal au cours du temps sont à l'origine de la baisse de productivité des variétés (McKey et al., 2012). Ce processus peut mener à la disparition localisée de certaines variétés, remplacées par d'autres, jugées plus performantes par les productrices. L'effet de ce processus sur la disparition des variétés de manioc et l'érosion de la diversité génétique de cette espèce a été mis en évidence dans d'autres pays, notamment au Bénin (Agre et al., 2015).
Enfin, nos résultats suggèrent que la proximité des centres urbains et des axes routiers renforce les dynamiques des portefeuilles variétaux des exploitations, ces transformations étant notamment plus marquées dans les villages d'Odziba, proches de Brazzaville, que dans les villages d'Oyo, éloignés des grands centres urbains. En effet, ces zones sont davantage soumises aux facteurs de changements socio-économiques tels que les programmes de développement agricole, de diffusion de matériel végétal ou l'apparition de filières de transformation.

\section{Conclusion}

Cette étude met en évidence le caractère fortement dynamique des pratiques de gestion de la diversité du manioc dans les exploitations familiales au Congo, ainsi que les différences considérables d'évolution de la composition variétale entre les villages et les sites. Elle montre également que les pressions biotiques semblent être un moteur majeur de l'évolution des portefeuilles variétaux. Ces résultats montrent la capacité des exploitations familiales à mobiliser la diversité variétale pour s'adapter aux modifications de leur environnement, et ouvrent de nouvelles perspectives de recherches concernant les modes d'approvisionnement en matériel végétal des exploitations familiales. L'ampleur des dynamiques de la diversité du manioc au Congo incite également à approfondir la réflexion concernant les stratégies pour sa conservation et sa valorisation. La grande diversité de ces dynamiques suggère que des stratégies visant à maintenir et à favoriser l'accès des exploitations à du matériel végétal fortement diversifié doivent être privilégiées, plutôt que celles visant à diffuser un nombre restreint de variétés d' ' élite ». En effet, la diversité des contextes auxquels les exploitations sont soumises fait qu'une variété adaptée à un contexte donné ne le sera pas forcément dans un autre. De ce fait, l'articulation entre conservation et valorisation du matériel doit être repensée pour favoriser d'une part la diffusion de la diversité maintenue dans les collections ex situ vers les exploitations familiales, et d'autre part pour enrichir régulièrement ces collections à partir de collectes in situ (Lebot 2014; Lebot et al., 2005 ; Seboka et Hintum, 2006).

\section{Matériel supplémentaire}

Tab. S1. Liste des analyses statistiques et librairies R utilisées. Tab. S2. Comparaison de l'abondance des variétés entre 2007 et 2012 dans les sites d'Oyo (haut) et Odziba (bas).

Le matériel supplémentaire est disponible sur https://www. cahiersagricultures.fr/10.1051/cagri/2017022/olm.

\section{Références}

Agre AP, Kouchade S, Odjo T, Dansi M, Nzobadila B, Assogba P, et al. 2015. Diversité et évaluation participative des cultivars du manioc (Manihot esculenta Crantz) au Centre Bénin. International Journal of Biological and Chemical Sciences 9(1): 388-408.

Altieri MA. 1999. The ecological role of biodiversity in agroecosystems. Agriculture, Ecosystems \& Environment 74(1): 19-31.

Boster JS. 1986. Exchange of varieties and information between Aguaruna manioc cultivators. American Anthropologist 88(2): 428-436. 
Caillon S, Lanouguère-Bruneau V. 2005. Gestion de l'agrobiodiversité dans un village de Vanua Lava (Vanuatu) : stratégies de sélection et enjeux sociaux. Journal de la Société des Océanistes 120-121(1): 129-148.

Chaudhary P, Gauchan D, Rana RB, Sthapit BR, Jarvis DI. 2004. Potential loss of rice landraces from a Terai community in Nepal: a case study from Kachorwa, Bara. Plant Genetic Resources Newsletter 137(1): 14-21.

Coomes OT, McGuire SJ, Garine E, Caillon S, McKey D, Demeulenaere E, et al. 2015. Farmer seed networks make a limited contribution to agriculture? Four common misconceptions. Food Policy 56(1): 41-50.

Elias M, McKey D, Panaud O, Anstett MC, Robert T. 2001. Traditional management of cassava morphological and genetic diversity by the Makushi Amerindians (Guyana, South America): perspectives for on-farm conservation of crop genetic resources. Euphytica 120(1): 143-157.

FAO. 2005. Congo: l'irrigation en Afrique en chiffres - Enquête AQUASTAT, $10 \mathrm{p}$.

FAO. 2006. Situation du manioc en République du Congo: état des lieux et perspectives. Rapport présenté par l'équipe nationale de coordination des activités sur le manioc au Congo, $26 \mathrm{p}$.

Kombo GR, Dansi A, Loko LY, Orkwor GC, Vodouhè R, Assogba P, et al. 2012. Diversity of cassava (Manihot esculenta Crantz) cultivars and its management in the department of Bouenza in the Republic of Congo. Genetic Resources and Crop Evolution 59(8): 1789-1803.

Labeyrie V, Thomas M, Muthamia ZK, Leclerc C. 2015. Seed exchange networks, ethnicity and sorghum diversity. Proceedings of the National Academy of Sciences 113(1): 98-103.

Lebot V. 2014. Strengthening smallholders' capacity to adapt to agroclimatic changes: optimization of root crops germplasm management and use. Journal of Root Crops 39(2): 5-12.

Lebot V, Ivancic A, Kuttolamadathil A. 2005. The geographical distribution of allelic diversity, a practical means of preserving and using minor root crop genetic resources. Experimental Agriculture 41(4): 475-489.

Legg JP, Owor B, Sseruwagi P, Ndunguru J. 2006. Cassava mosaic virus disease in East and Central Africa: epidemiology and management of a regional pandemic. Advances in Virus Research 67(1): 355-418.

Legg JP, Jeremiaha SC, Obiero HM, Maruthi MN, Ndyetabula I, Okao-Okuja G, et al. 2011. Comparing the regional epidemiology of the cassava mosaic and cassava brownstreak virus pandemics in Africa. Virus Research 159(2): 161-170.

Letourneau DK, Armbrecht I, Rivera BS, Lerma JM, Carmona EJ, Daza MC, et al. 2011. Does plant diversity benefit agroecosystems? A synthetic review. Ecological Applications 21(1): 9-21.

Mabanza J. 1992. La sélection et l'amélioration du manioc au Congo : acquis et perspectives. Brazzaville (Congo) : CERAG/DGRST, 127 p.

McGuire S, Sperling L. 2016. Seed systems smallholder farmer use. Food Security 8(1): 179-195.

McKey D, Elias M, Pujol B, Duputié A, Delêtre M, Renard D. 2012. Maintien du potentiel adaptatif chez les plantes domestiquées à propagation clonale. Leçons de gestion par les cultivateurs de manioc amérindiens. Revue d'Ethnoécologie 1. Disponible sur http://ethnoecologie.revues.org/741.

Mvila AC, Ntawuruhunga P, Bembe PA, Obambi M. 2003. La mosaïque africaine du manioc en République du Congo: distribution et importance de la maladie. in: 13th symposium of International Society for Tropical Root Crop (ISTRC), ArushaTanzania, November 10-14th 2003.

Oksanen J, Blanchet FG, Kindt R, Legendre P, Minchin PR, O'Hara RB, et al. 2013. Package 'vegan'. Community ecology package, version 2. 2-0.

Olsen KM, Schaal BA. 1999. Evidence on the origin of cassava: phylogeography of Manihot esculenta. Proceedings of the National Academy of Sciences 96(10): 5586-5591.

Otabo FR, Labeyrie V, Duval MF, Mabanza J, Mialoundama F. 2016. Diversité variétale de manioc sur la base des nominations vernaculaires des agriculteurs dans 4 bassins de production (Hinda, Loudima, Odziba et Oyo) en République du Congo. Journal of Applied Bioscience 104(1): 9932-9941.

Peroni N, Hanazaki N. 2002. Current and lost diversity of cultivated varieties, especially cassava, under swidden cultivation systems in the Brazilian Atlantic Forest. Agriculture, Ecosystems \& Environment 92(2): 171-183.

Pham JL, Morin SR, Sebastian LS. 2002. Rice, farmers and genebanks: a case study in the Cagayan valley, Philippines. In Engels JMM, Bamanatha Roa V, Brown AHD, Jackson MT, eds. Managing plant genetic resources. New York: CABI, pp. 149-159.

Pinton F, Emperaire L. 2001. Le manioc en Amazonie brésilienne : diversité variétale et marché. Genetic Selection and Evolution 33 (1): 491-512.

Pujol B, David P, McKey D. 2005. Microevolution in agricultural environments: how a traditional Amerindian farming practice favours heterozygosity in cassava (Manihot esculenta Crantz, Euphorbiaceae). Ecology Letters 8(2): 138-147.

Ratnadass A, Fernandes P, Avelino J, Habib R. 2012. Plant species diversity for sustainable management of crop pests and diseases in agroecosystems: a review. Agronomy for Sustainable Development 32(1): 273-303.

Rossi JP. 2011. Rich: an R package to analyse species richness. Diversity 3(1): 112-120.

R Core Team. 2015. R: a language and environment for statistical computing. R Foundation for Statistical Computing, Vienna (Austria). Available from https://www.R-project.org/.

Sardos J, McKey D, Duval MF, Malapa R, Noyer JL, Lebot V. 2008. Evolution of cassava (Manihot esculenta Crantz) after recent introduction into a South Pacific Island system: the contribution of sex to the diversification of a clonally propagated crop. Genome 51(11): 912-921.

Scherer R, Pallmann P. 2014. Simboot: simultaneous inference for diversity indices. $R$ package version $0.2-5$.

Seboka B, Hintum VT. 2006. The dynamics of on-farm management of sorghum in Ethiopia: implication for the conservation and improvement of plant genetic resources. Genetic Resources and Crop Evolution 53(7): 1385-1403.

Shannon CE. 1948. A mathematical theory of communication. Bell System Technical Journal 27(1): 379-423.

Citation de l'article : Otabo FR, Labeyrie V, Duval M-F, Mabanza J, Mialoundama F. 2017. Dynamiques temporelles de la diversité variétale du manioc (Manihot esculenta Crantz) dans deux bassins de culture en République du Congo. Cah. Agric. $26: 45004$. 